\title{
Do we need yet another journal?
}

\author{
Vincent T DeVita, Jr
}

We live in the most exciting time in the history of cancer medicine. The molecular revolution has reached the bedside. With this excitement comes an enormous burden physicians must bear on behalf of their patients. Physicians must select, gather and read the most relevant literature of the day, to stay current and to keep pointed toward the future, intellectually.

The goal of Nature Clinical Practice Oncology, one of a news series of clinical journals from the publishers of Nature, is to aid cancer physicians in doing just that. Nature Publishing Group has unmatched resources in this arena. I am pleased and proud to work with such a fine organization, with its reputation for quality, and with our internationally known Advisory Board, to try to accomplish this task.

This journal has some unique editorial features that will ease your workload and help you interpret and put into practice the enormous amount of published research. The journal acts as a filter, identifying not just the obvious research journals but also journals that may only occasionally publish original research relevant to your specialty. Nature Clinical Practice Oncology will also promote the chronology of medical research and application by reporting on it, interpreting it and offering an informed opinion on it over a period of time.

The journal includes several types of article. Research Highlights are short summaries of relevant articles published in other journals. Practice Points are a unique feature of the journal that document and interpret influential research papers published elsewhere. They consist of a structured synopsis written in-house and a commissioned commentary written by an expert in the field, which puts the original article into context. The Practice Point itself summarizes how the original article should affect the way you treat your patients.

\section{This journal \\ has some \\ unique editorial \\ features that \\ will ease your \\ workload \\ and help you \\ interpret and \\ put into practice \\ the enormous \\ amount of \\ published \\ research}

VT DeVita, Jr is the Editor-in-Chief of Nature Clinical Practice Oncology.

Competing interests

The author declared he has no competing interests.

www.nature.com/clinicalpractice doi: $10.1038 / n c p o n c 0027$
Viewpoints are opinion articles, in which authors discuss or debate topical subjects in oncology. Review articles, of which there are several types, are timely, authoritative overviews of developments in the specialty. Therapy Insights provide information about medical conditions being managed by physicians in other specialties. Each month a leading specialist will write about how their specialty impacts on oncology. Drug Insights review relevant information about a drug class; Mechanisms of Disease provide insights into the science underlying cancer. Technology Insights focus on advances in treatment and diagnostic technologies and equipment, such as imaging techniques and technology-assisted surgery. Primer articles provide simple, jargon-free explanations of areas essential to the understanding of clinical research by non-trialists, such as statistical methods and good clinical practice. Finally, Case Studies describe interesting studies of patients presenting with unusual symptoms or in unusual circumstances, with a thorough discussion of diagnosis and management. You also have the opportunity to earn Category 1 CME credits through the journal's partnership with Vanderbilt University Medical School.

All content will undergo peer-review, with two exceptions: the Editorial and the Research Highlights. Content is editorially independent; authors are asked to state any professional or financial situations that may be perceived as causing a conflict of interest with respect to integrity of content.

The journal's international Advisory Board shares this vision, and is integral in acheiving the highest quality and most useful publication available to oncologists. They will ensure that all aspects of oncology are covered.

I believe that Nature Clinical Practice Oncology will improve your knowledge base and help you treat your patients optimally. 